\title{
Investigation of Low-cost Cash Register Management System with Electronic Scale and Cloud Connection
}

\author{
Zhiwen Cai, ${ }^{1,2}$ Jingying Ke, ${ }^{3 *}$ Cheng-Fu Yang, ${ }^{4, * *}$ and Man-Ying Lee ${ }^{6}$ \\ ${ }^{1}$ School of Management, Xiamen University, Xiamen, 361024, Fujian Province, China \\ ${ }^{2}$ School of Economics and Management, Xiamen University of Technology, \\ Xiamen, 361021, Fujian Province, China \\ ${ }^{3}$ Business School, Xiamen Institute of Technology, Xiamen, 361024, Fujian Province, China \\ ${ }^{4}$ Department of Chemical and Materials Engineering, National University of Kaohsiung, Kaohsiung 811, Taiwan \\ ${ }^{5}$ Department of Aeronautical Engineering, Chaoyang University of Technology, Taichung 413, Taiwan \\ ${ }^{6}$ Department of Industrial Economics, Tamkang University, Taipei Tamsui 251, Taiwan
}

(Received October 21, 2020; accepted January 27, 2021)

Keywords: embedded system, point of sale (POS), remote monitoring, cloud service, cash register management system

Electronic scales and cash registers play a universal role in transactions at the checkout systems of businesses. To reduce the number of errors occurring at the checkouts of buffet restaurants using electronic scales, a novel electronic scale measuring system is designed and investigated in this study. This designed checkout system was constructed using a Tibbo embedded chip in the cash register management system with a cloud connection. This proposed system is different from the point of sale (POS) system: it can obtain all the information of sale items at the same time, and it has the advantages of a low cost and simple operation. Furthermore, the system can transfer each trading record to be saved in a remote server through a cloud transfer service, and restaurants using this system can construct an effective accounting management mechanism. A weighed quantity is transformed into a digital quantity and sent to the cloud system by the embedded system. As compared with traditional, expensive, and large POS systems, the proposed system has higher measuring accuracy and is less affected by staff errors. The low-cost cash register management system with a cloud connection will be a competitive digital product for future commercial business activities.

\section{Introduction}

The catering industry uses an electrical scale and cash register for transactions as a normal business behavior in today's society. Eating a meal in a buffet restaurant is one of the most common dining modes for fast-food fans because they can choose their favorite foods with different combinations of ingredients, and customers can determine the size of their meals according to their appetite. A common pricing method in buffet restaurants is to weigh the food selected by customers and charge a price based on the weight. Then the money to be paid by the customers is shown on a liquid crystal display (LCD) as a reference for customers and

\footnotetext{
*Corresponding author: e-mail: kejingying@xit.edu.cn

${ }^{* *}$ Corresponding author: e-mail: cfyang@nuk.edu.tw

https://doi.org/10.18494/SAM.2021.3170
} 
checkout staff. ${ }^{(1-7)}$ A point of sale (POS) system is used by businesses to calculate the amounts of goods purchased by customers and their prices and prepare an invoice for the customers, and it can also indicate the options by which the customers can make their payment. ${ }^{(8-10)}$ The traditional keyboard-type cash registers need checkout staff to type the price of each commodity. In the checkout process, the staff may make some errors in the amount because they are busy, and these errors will cause operation losses and are not easy to overcome by accounting management. ${ }^{(11)}$ If we can design a system with a smooth checkout process and an accounting management mechanism, we can avoid such problems and also can make an effective management mechanism for the accounting of revenue. ${ }^{(12,13)}$

When there are more customers, however, the likelihood of typed errors increases, and dealing with such errors may further increase the length of a queue of waiting customers. So far, the most efficient cash registers have an automatic reading function that is integrated with optical scanning instruments for the cashier to scan one-dimensional bar codes into the system. The obtained data can be saved in a storage controller, and then the cash register gets the required information and data for analyses and calculation. ${ }^{(14,15)}$ If the checkout process is digitized, the errors caused by human typing can be reduced, the time for the checkout processes can be reduced, and the training process for staff can be simplified. Then, the speed and efficiency of checkout processes can be enhanced and a digitized process can be used as an alternative to human cashiers.

Currently, there are still many traditional buffet restaurants with non-fixed or uncertain conditions in their commodity transactions, who use the unit price and weight of items as the pricing standard. The cash register management system proposed in this study is an electronic scale system with an embedded system and chip as the hardware and a cloud connection as the software; thus, it is a packaging and assembly system. Because the weight of items fluctuates in a buffet, it is not appropriate to use a large amount of bar codes for digitization management. If we can solve the problems associated with a variable product weight, then we can still use a high-efficiency management system in digitization checkout processes while enhancing the efficiency of traditional industries using weight-based pricing. The novelty of the proposed system is that a low-cost Tibbo embedded system is used as the platform with a digital cashier management process as the core. As compared with traditional expensive and large POS systems, the proposed system has higher measuring accuracy with fewer errors caused by the staff. ${ }^{(16-19)}$ The proposed system can not only be used as a cash register management system with high efficiency, it can also reduce the production cost of cash register management systems.

\section{System Architecture and Hardware Planning}

\subsection{Source and structure of EM1000 embedded system}

In this study, we used an embedded system and chip, which uses a Tibbo operating system (TiOS) as the firmware main core (EM1000) and has the functions in Table 1. The EM1000 includes 10/100 Ethernet, four serial ports, flash memory, and 54 universal pins for input and output (GPIO), 
Table 1

Specification of Tibbo EM1000 embedded system.

\begin{tabular}{|c|c|c|c|}
\hline Function & Specification & Function & Specification \\
\hline Frequency & $88 \mathrm{MHz}$ & RTC power & Onboard supercapacitor \\
\hline EEPROM & 2 KByte & PLL control & Hardware/Software \\
\hline Flash memory & 1024 KByte & Reliable circuit & Power-on/Brown-out \\
\hline Ethernet & 10/100 Base/T & Power & $230 \mathrm{~mA} / 3.3 \mathrm{~V}$ \\
\hline GPIO lines & 54 (8 Interrupt lines) & Dimensions & $38.4 \times 28.4 \times 5.5 \mathrm{~mm}^{3}$ \\
\hline Serial ports & 4 (Up to $921600 \mathrm{bps})$ & & \\
\hline
\end{tabular}

Table 2

Specification core functions and scope of applications of EM1000 system.

\begin{tabular}{|c|c|c|c|}
\hline Core functions & Scope of applications & Core functions & Scope of specification \\
\hline Sock & Web server connection & Stor & Execution block of main program \\
\hline Ser & Electronic scale, keyboard, LCD & Pat & Trigger event of LED control \\
\hline $\mathrm{IO}$ & $\begin{array}{l}\text { Switch control of electronic cash } \\
\text { register }\end{array}$ & Beep & System warning sound \\
\hline $\mathrm{Kp}$ & Input signal of digital keyboard & Button & Button trigger switch \\
\hline Rtc & $\begin{array}{l}\text { Functions of system time and standby } \\
\text { power supply }\end{array}$ & Sys & Reset and system clock \\
\hline $\mathrm{Fd}$ & $\begin{array}{l}\text { Block for recording customer } \\
\text { information }\end{array}$ & & \\
\hline
\end{tabular}

and it also provides a network access function, the external connection of a liquid crystal display (LCD) device, a number keyboard, an RS 232 module, and the application of an on/off switch controller.

\subsection{Supporting functions of EM1000 embedded system}

The embedded chip can provide many programmable functions, for example, the communication and controller of Ethernet, communication with an RS 232 series port, keyboard input, an LED controller, a buzzer, signal input/output and interruption, memory, file management, a web server, and buttons for remoting hardware. The hardware and functions can be used to implement a low-cost and cloud cashier management system. The application functions of the proposed system are shown in Table 2.

\subsection{Peripheral planning of EM1000 embedded system}

The main applications of the proposed system are weighing and pricing, for which the embedded system is the basic platform, and we also need an electrical scale that can output the weight. For this, an LPWN-7515 table-type electrical scale with precision of $0.5 \mathrm{~g}$ (abbreviated to "electrical scale") is used for weighing. The weight data can be transmitted to the embedded platform during the operational processes through the RS 232 transmission module. The embedded platform is connected to the different external modules, including the LCD module, digitalization keyboard, on/off control module, and internal connection module, thus comprising the low-cost cash register management system with a cloud connection shown in Fig. 1. 


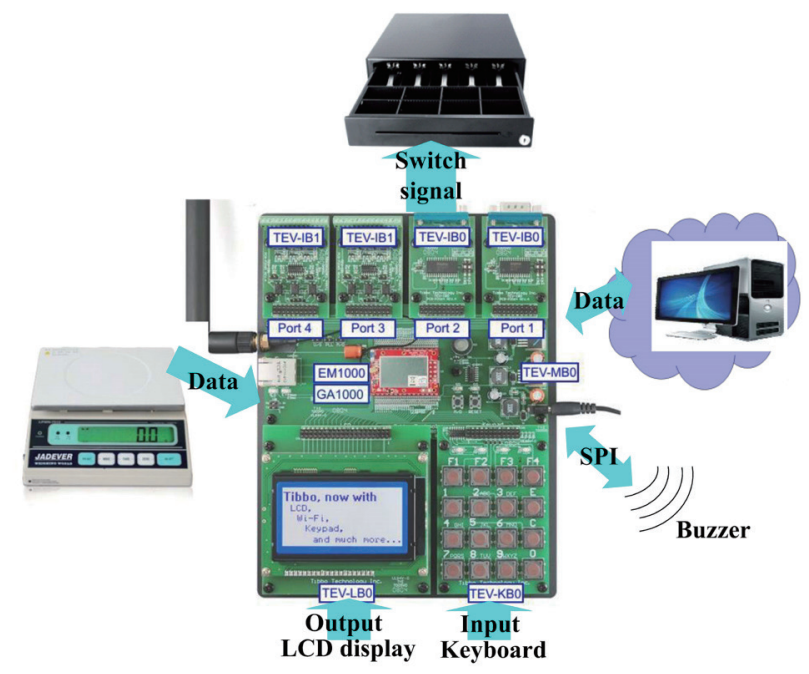

Fig. 1. (Color online) Planning diagram of system applications based on EM1000.

Table 3

System functions and scope of applications of EM1000 system.

\begin{tabular}{lcllc}
\hline Keyboard symbol & \multicolumn{1}{c}{ Function of applications } & & Keyboard symbol & Function of specification \\
\hline F1 & Function of value-added items & & C & Delete data \\
F2 & Display current total revenue & & $0-9$ & Input number \\
F3 & Calculation function of change bills & M/D & Save data/pop-up cash register \\
F4 & Confirm sum of current transaction & RES & Reset system \\
E & Calculate price after items are weighed & &
\end{tabular}

\section{Functions and Applications of Proposed System}

\subsection{Function design of EM1000 system}

For effective operation of the cash register management system, we defined functions for some of the buttons on the keyboard as listed in Table 3. The operation modes and practical application steps of the fabricated system are shown in Figs. 2-6. First, as shown in Fig. 2(a), the electrical scale weighs the item, then, as shown in Fig. 2(b), the EM1000 embedded platform calculates the cost of the weighed item. Next, the unit price of additional items to be purchased is keyed in and the LCD shows the new amount [Fig. 3(a)]. If necessary, a function for giving change from a large bill is started up and the LCD shows the amount of change [Fig. 3(b)]. The third process is to save the information of the total amount as shown in Figs. 4(a) and 4(b). We press function key F4, then the information of the total amount is saved, and when we press function key F2, we can check the current total revenue. We can also check on the website for the status of each item of revenue, as shown in Fig. 5(a), and when the staff presses function key $M / D$, the system automatically saves each item of revenue into the database of the total revenue, as shown in Fig. 5(b). Finally, as shown in Fig. 6(a), as the amount of each transaction 


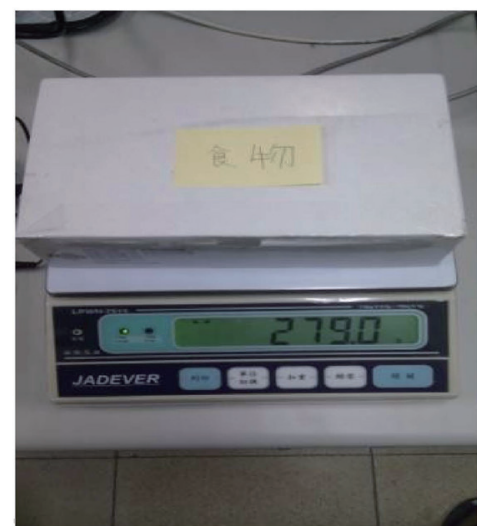

(a)

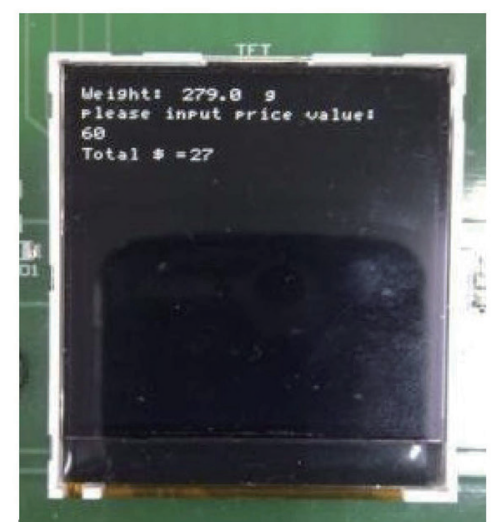

(b)

Fig. 2. (Color online) (a) Weighing of item and (b) cost of the weighed item.

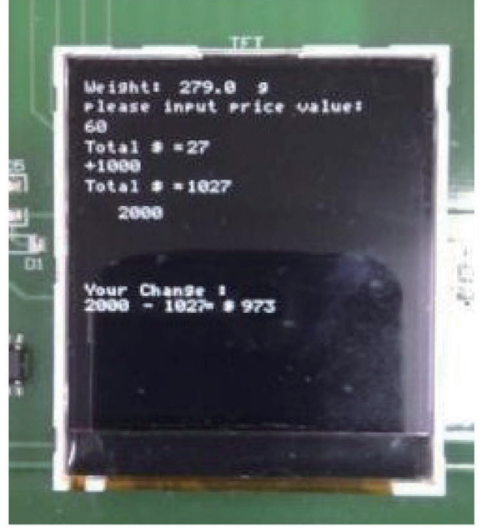

(a)

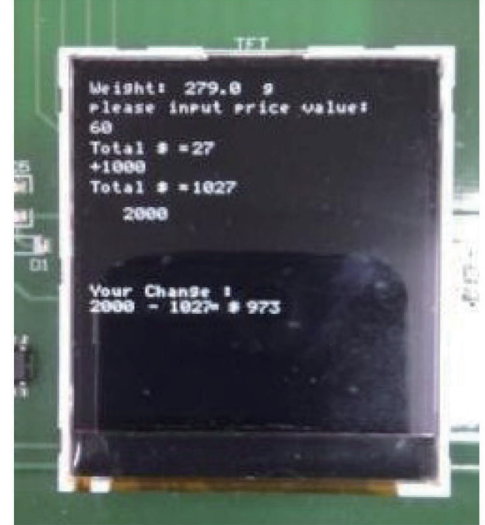

(b)

Fig. 3. (Color online) (a) When extra items are to be purchased, the unit price of the extra items is keyed in and the LCD shows the new amount. (b) The change function starts up and the LCD shows the amount of change.

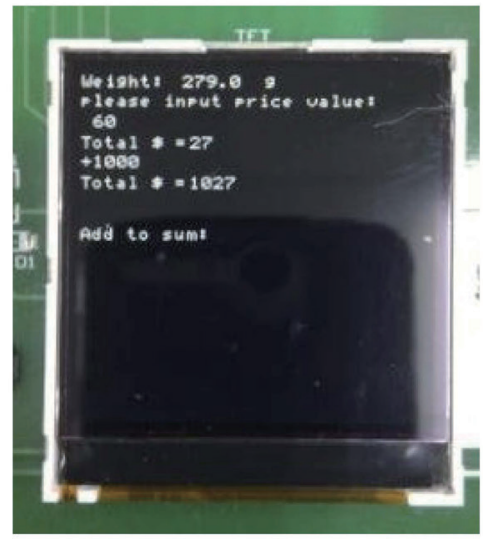

(a)

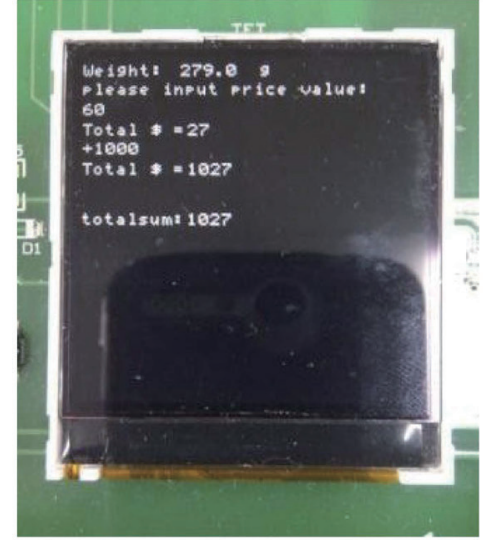

(b)

Fig. 4. (Color online) (a) Function key F4 is pressed to save the information of the amount and (b) function key F2 is pressed to check the current total revenue. 


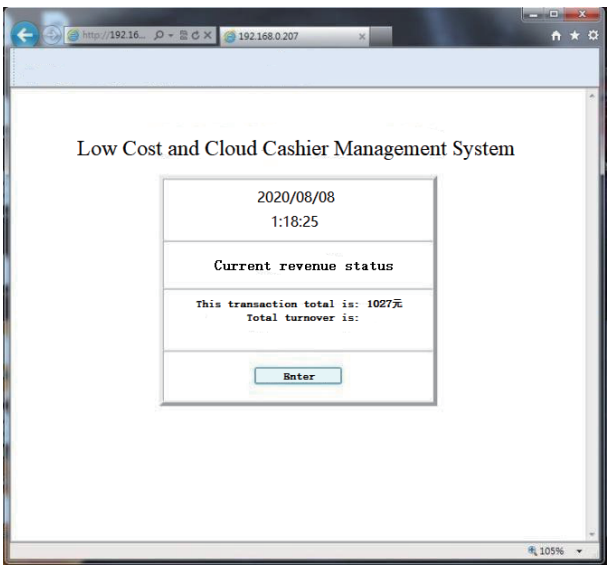

(a)

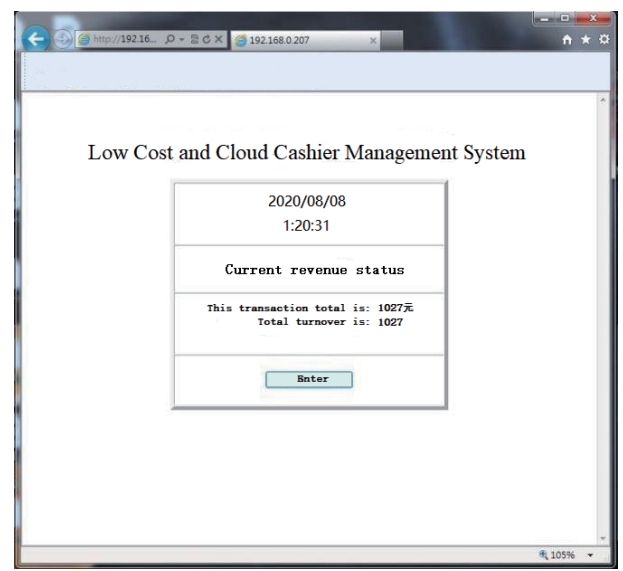

(b)

Fig. 5. (Color online) (a) The status of each item of revenue can be checked on the website and (b) function key $\mathrm{M} / \mathrm{D}$ is pressed to save each item of revenue into the database of total revenue.

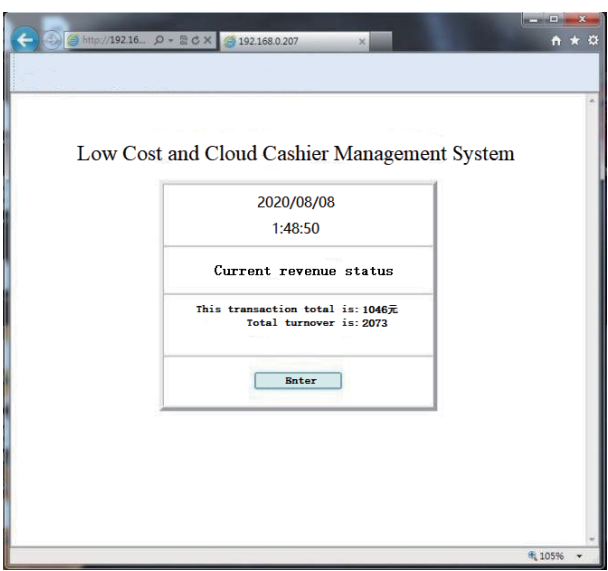

(a)

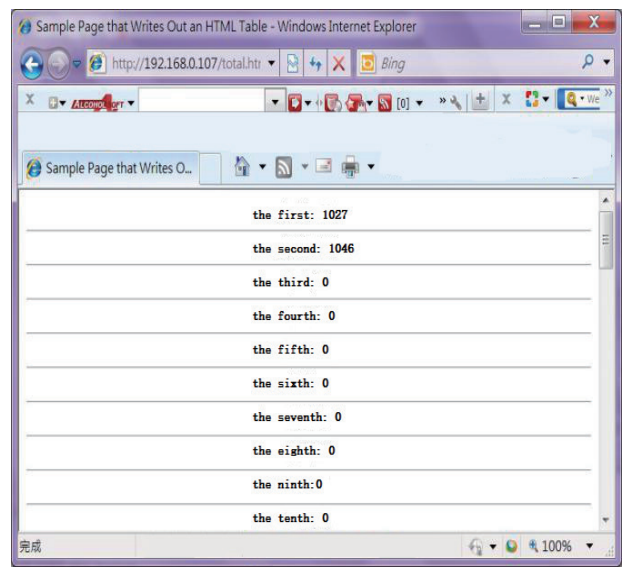

(b)

Fig. 6. (Color online) (a) The EM1000 embedded system saves the amount of each transaction after its checkout and (b) an employee can remotely survey the account for each item and the total revenue on the website.

is checked, the EM1000 embedded system automatically adds it to the total revenue. When a company employee wants to check the account for each item and the total revenue, he or she can use a remote system to survey in detail the information of each transaction on the website, as shown in Fig. 6(b).

\subsection{Signal flow design of EM1000 system}

Now that we have explained the definitions and functions of the hardware, we discuss the reception conditions as the software references of the cash register to design a system for checkout and management processes. This process is the core of the operation of the proposed software, and the designed checkout and management processes are shown in Fig. 7. 


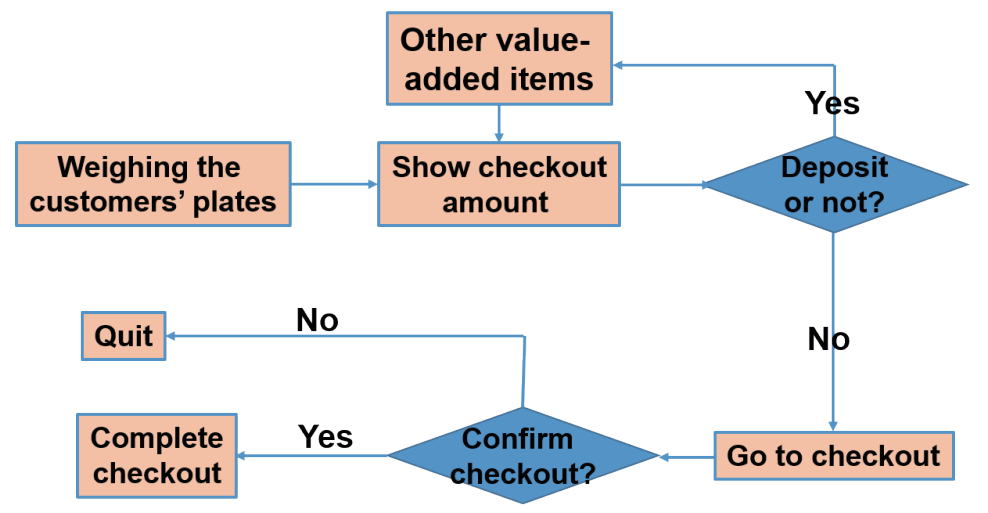

Fig. 7. (Color online) Flow chart of checkout in the proposed system.

\subsection{Application process of EM1000 system}

Figure 8 shows the flow chart for the low-cost cash register management system with an electrical scale and a cloud connection. As the system is booting up, the red LED turns on. The data's input and connection for the cash register management system with the electrical scale are through the serial ports of the RS 232 module. As the customers put their meals or the weighed food on the electrical scale, the weight information is transmitted to the EM1000 platform and shown on the LCD. First, the checkout staff needs to input the unit price of the priced meal and presses key E for the calculation, then the price for the current weight is shown on the LCD of the embedded system. If the customer wants to purchase more food, the checkout staff presses function key F1 and then the LCD shows the new amount. If the customer or staff finds an error, the staff can press key C, then the amount shown on the LCD goes back to its initial state and the electrical scale is used to reweigh the meal and calculate the price to the satisfaction of the customer and staff.

If the customer does not want to purchase more food, the staff presses function key F4 to undergo the processes of verification and data saving. The cost of the transaction is saved into the memory by pressing function key F4, and the LCD shows the amount on the display. After the customer and staff have confirmed the amount, the staff presses function key M/D to open the cash register's cashbox to process the checkout and give change to the customer (at that time, a buzzer sounds three times). If the customer hands over a large bill and the amount of change needs to be calculated, the EM1000 platform can provide a convenient function for calculating the change. The staff types the size of the bill and presses function key F3 and the LCD shows the amount of change to help the staff avoid errors when giving change. While the above processes are performed, the embedded system transfers the customer's information to the company website. When a company member wants to know the revenue at any time, they just press function key F2 on the EM1000 platform and then the revenue is shown on the LCD. If a full report of the received amount is required, the EM1000 platform also provides the information for each transaction, which is saved on the website. 


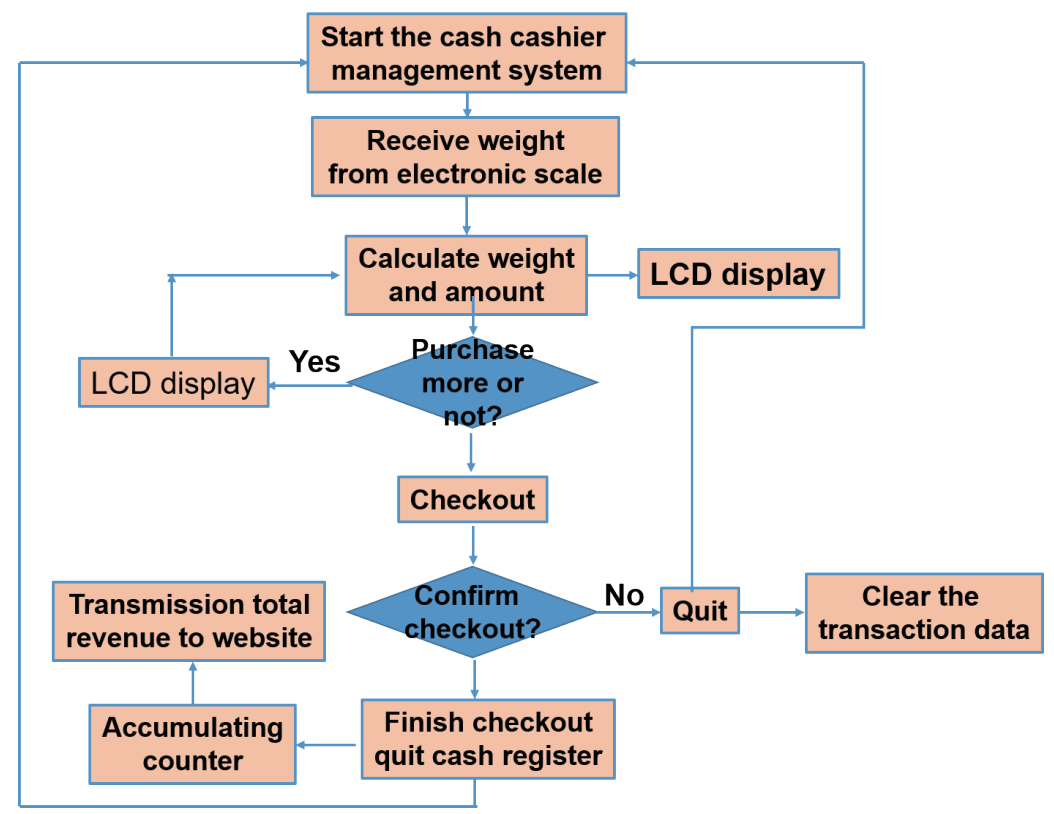

Fig. 8. (Color online) Flow chart for electronic scale for low-cost cash register management system with cloud connection.

\section{Innovation and Design Considerations}

The innovative feature of the EM1000 embedded platform proposed in this study is that the cash register management system is digitized and enables convenient accounting management; thus, the number of errors made by checkout staff will decrease even they use the manual calculation mode for checkout. A traditional large POS system usually uses complicated application programs, and the checkout efficiency is reduced by communication and connection delays between the POS host system and other equipment. The design of the proposed system eliminates the complex operating procedures and uses the actual cash register processes as the basis of the design. The advantages of the EM1000 embedded system are that it does not need a complicated operation system to complete the communication with other peripheral devices and perform the calculation. The problem of system delays will also not happen because the EM1000 embedded system does not use large application programs. As a result, the proposed system can not only provide a high-efficiency cash register management system but should also have a lower production cost.

\section{Practical Contribution and Comparison of Two Systems}

\subsection{Practical contribution}

The biggest contribution of the novel EM1000 embedded platform is its low cost and simple operation, enabling checkout staff to quickly become familiar with its operation. This 
Table 4

Comparison of electronic scales with cash register management system and traditional POS system.

\begin{tabular}{lcc}
\hline Function & Traditional POS system & $\begin{array}{c}\text { Low-cost and cloud cash register } \\
\text { management system }\end{array}$ \\
\hline Price & Higher & Lower \\
\hline $\begin{array}{l}\text { Centralized management } \\
\text { in cloud and web monitoring }\end{array}$ & No & Yes \\
\hline Automatic pricing & Yes & Yes \\
\hline Operational convenience & Complicated & Simple \\
\hline System in module & No & Yes \\
\hline
\end{tabular}

system can solve the errors caused by the manual operation and provide an easy method for solving problems with accounting management. Thus, this system can replace an expensive and complicated POS restaurant management system, and it can use a cloud server for the centralized management of a chain of restaurants or use the company website to remotely obtain the current revenue. The proposed system has the functions of historical data tracking and a management mechanism for comparing accounting data, which can help restaurants avoid incurring operating losses.

\subsection{Comparison and analyses of two systems}

The purpose of this research is to develop a system for buffet restaurants with the functions of weighing and pricing. The large POS systems traditionally used for weighing and pricing in restaurants are expensive and have the shortcomings of high power consumption and lack of suitability for the environments of traditional catering establishments. The proposed embedded system has low power consumption and is cheaper than POS systems. This system has a fully developed modular chip that can be integrated with any type of electrical scale to realize a next-generation cloud service system. As shown in Table 4, the proposed low-cost embedded platform is applied in the weighing and pricing industries, and its functions can replace those of a complicated POS system. In the future, if the capacity of flash memory increases or an external secure digital (SD) memory card is connected, this system can provide more complete checkout information, for example, information on the staff. Such information can be saved in the terminal system of the EM1000 embedded platform and give the catering manager the ability to synchronize restaurant management through the website or using a smartphone via the cloud.

\section{Conclusions}

In this study, we proposed a low-cost cash register management system using the embedded firmware system and chip of Tibbo Technology Ltd., which can be monitored on the cloud. Whereas a traditional large POS system uses complicated application programs and communication and connection delays occur between the POS host system and other equipment, the EM1000 embedded system does not need a such complicated operation system to complete the calculation functions and communicate with other peripheral devices. The proposed system 
reduces the number of errors caused by the manual operation of checkout staff and provides an easy method for staff to solve problems with accounting management. Purchase information can be saved in the terminal system of the EM1000 embedded platform and enable a catering manager to synchronize the restaurant's management via the website or a smartphone via the cloud.

\section{Acknowledgments}

We would like to thank Tibbo Technology Co., Ltd. for their kind support of the EM1000 system. This work was also supported by project numbers MOST 109-2622-E-390-001-CC3 and MOST 109-2221-E-390-023; the Youth Project of the Humanities and Social Sciences of the Ministry of Education under Grant 18YJC630140, China; and Social Science Fund of Fujian Province, China (FJ2020B023).

\section{References}

1 R. Gu, X. Ge, and Y. Lang: Proc. 2017 Int. Conf. Sensing, Diagnostics, Prognostics, and Control (SDPC, 2017) 670.

2 H. Zhan, P. Zhou, and X. Xiong: Proc. 2017 Int. Conf. Computer Technology, Electronics and Communication (ICCTEC, 2017) 770.

3 G. Xie, G. Zeng, X. Xiao, R. Li, and K. Li: IEEE Trans. Parallel Distrib. Syst. 28 (2017) 3426.

4 W. J. Wang and C. H. Lin: IEEE Trans. Very Large Scale Integr. VLSI Syst. 24 (2016) 266.

5 G. Xie, Y. Chen, R. Li, and K. Li: IEEE Trans. Ind. Inf. 14 (2018) 2418.

6 A. Korać, V. Ilic, N. Kovacic, D. Samardzija, and B. Pavkovic: 2017 13th Int. Conf. Advanced Technologies, Systems and Services in Telecommunications (TELSIKS, 2017) 431.

7 Q. Liu, Q. J. Zhang: IEEE Trans. Very Large Scale Integr. VLSI Syst. 24 (2016) 2062.

8 A. Dey, S. Jain, and S. Nandi: 2019 Int. Conf. Recent Advances in Energy-efficient Computing and Communication (ICRAECC, 2019) 1.

9 B.A.S. Sarrafpour, R. Del Pilar Soria Choque, B. Mitchell Paul, and F. Mehdipour: 2019 IEEE Int. Conf. Dependable, Autonomic and Secure Computing, on Pervasive Intelligence and Computing, on Cloud and Big Data Computing, and on Cyber Science and Technology Congress (DASC/PiCom/CBDCom/CyberSciTech, 2019) 493.

10 C. Samaranayake, R. K. Achchige, T. Shanaz, A. Ranasinghe, and A. Senarathne: 2019 Int. Conf. Advancements in Computing (ICAC, 2019) 109.

11 K. N. Anand, V. S. Prasad, and R. Bhandari: 2018 3rd IEEE Int. Conf. Recent Trends in Electronics, Information \& Communication Technology (RTEICT, 2018) 1336.

12 D. Kozlov, L. Druzhinina, N. Sadovnikova, and D. Petrova: 2018 Eleventh Int. Conf. Management of Largescale System Development (MLSD, Moscow, 2018) 1.

13 A. Ampuni, S. Fonataba, A. Fitrianto, and G. Wang: 2019 Int. Conf. ICT for Smart Society (ICISS, 2019$) 1$.

14 J. Harpanahalli, K. Bhingradia, P. Jain, and J. Koti, 2020 Fourth Int. Conf. Computing Methodologies and Communication (ICCMC, 2020) 87.

15 Y. Chen, W. Sun, N. Zhang, Q. Zheng, W. Lou, and Y. T. Hou: IEEE Trans. Inf. Forensics Secur. 14 (2019) 183.

16 A. Brahman, S. Sassani, and D. P. Rosario: 24th IEEE Pacific Rim Int. Symp. Dependable Computing (PRDC 2019) 1.

17 C. H. Hsia: IEEE Sens. J. 18 (2018) 790.

18 C. H. Chen, C. Y. Chen, C. H. Hsia, and G. X. Wu: Inter. J. Web Servi. Res. 14 (2017) 44.

19 H.L. Shieh and Y. C. Liao: 2011 Int. Conf. Machine Learning and Cybernetics (ICMLC 2011) 528. 